\title{
Dual Levitated Coils For Antihydrogen Production
}

\author{
J.D. Wofford and C.A. Ordonez
}

Department of Physics, University of North Texas, Denton, Texas

\begin{abstract}
Two coaxial superconducting magnetic coils that carry currents in the same direction and that are simultaneously levitated may serve for antihydrogen plasma confinement. The configuration may be suitable for use by a collaboration at the CERN Antiproton Decelerator facility to test fundamental symmetries between the properties of hydrogen and antihydrogen. Nested Penning traps are currently used to confine recombining antihydrogen plasma. Symmetry studies require the production of sufficiently cold antihydrogen. However, plasma drifts within nested Penning traps can increase the kinetic energy of antiprotons that form antihydrogen atoms. Dual levitated coils may serve to confine relatively large, cold, dense non-drifting recombining antihydrogen plasmas. A minimum-B magnetic field that is produced by the coils could provide for atom trapping. A toroidal plasma is confined between the coils. High density plasmas may be possible, by allowing plasma pressure to balance mechanical pressure to keep the coils apart. Progress is reported on theoretical and experimental efforts. The theoretical effort includes the development of a classical trajectory Monte Carlo simulation of confinement. The experimental effort includes levitation of a $\mathrm{NdFeB}$ permanent ring magnet, which produces a magnetic field that is qualitatively similar to the field that would be produced by the two coaxial superconducting magnetic coils. Liquid-nitrogen-cooled Bi-2223 high-temperaturesuperconducting components, with a critical temperature of $108 \mathrm{~K}$, were used to levitate the ring magnet. An issue concerning keeping the plane of the levitated ring horizontal is discussed.
\end{abstract}

Keywords: CERN AD, antihydrogen, recombination, plasma, confinement

PACS: $37.10 \mathrm{Ty}, 52.55 \mathrm{Hc}, 52.65 \mathrm{Cc}, 52.65 \mathrm{Pp}$

\section{INTRODUCTION}

A plasma confinement concept that uses a pair of coaxial superconducting magnetic coils may be suitable for use by a collaboration at the CERN Antiproton Decelerator facility to test fundamental symmetries between the properties of hydrogen and antihydrogen. Currently, nested Penning traps are used to confine recombining antihydrogen plasma [1-3]. Symmetry studies require the production of sufficiently cold antihydrogen. Plasma drifts within the traps can increase the kinetic energy of the antiprotons that form antihydrogen atoms [4]. The dual-coil system proposed here may provide the conditions needed to confine relatively large, cold, dense, nondrifting, recombining antihydrogen plasmas.

Plasma devices that use a single levitated coil have been built and operated successfully. Among these are the Levitated Dipole Experiment (LDX) [57] and Ring Trap-1 (RT-1) [8-10]. In these devices, a levitation coil produces a magnetic field that serves to levitate and stabilize the levitated coil. An optical array detects any deviation from the equilibrium position. This signal is fed to an amplifier, which drives the levitation coil. The levitation coil applies the force necessary to keep the levitated coil in an equilibrium position. In the case of complete loss in control of the levitated coil, the devices have fail-safes that catch the levitated coil to prevent damage $[5,9]$.

It has already been demonstrated that levitation and stabilization of one coil can be achieved using an active feedback system. However, this type of feedback may not be practical for systems that are levitating multiple coils [11]. A more practical system to use for the case of multiple levitated coils is a passive feedback system, in which no motion detection or externally controlled forces are required.

Superconducting materials can provide a passive feedback system, so long as a few logistical issues can be overcome. A configuration of superconductors is needed such that the accessibility to the plasma being confined is not hindered too greatly. There is also the problem of keeping a superconductor below its critical temperature, which depends on the material used.

The present paper describes a simulation of the confinement of antihydrogen plasma particles within the dual-coil system. Also presented is an experimental demonstration of the use of superconductors as a passive feedback system. 


\section{SIMULATION OF A DUAL-COIL PLASMA CONFINEMENT SYSTEM}

A circular current loop of infinitesimal thickness is used here as a model for a multiple- or single-turn coil of current-carrying wire. The axial and radial magnetic field components for the system, with the central symmetry axis aligned with the z-axis of a cylindrical coordinate system, are non-zero. The azimuthal magnetic field component is zero, because a circular current loop is cylindrically symmetric. By placing two current carrying loops next to each other coaxially, there is a circular null line from which the magnitude of the magnetic field increases in all directions. Figure 1 illustrates dual levitated coils and the magnetic field lines. A magnetic field line that passes close to the null circle is a closed field line, which does not intersect a solid material, provided the two loops are levitated. Particles that follow such field lines will return to the vicinity of the null circle. Plasma confined in the vicinity of the null circle is describable as being confined by a magnetic mirror with closed field lines.
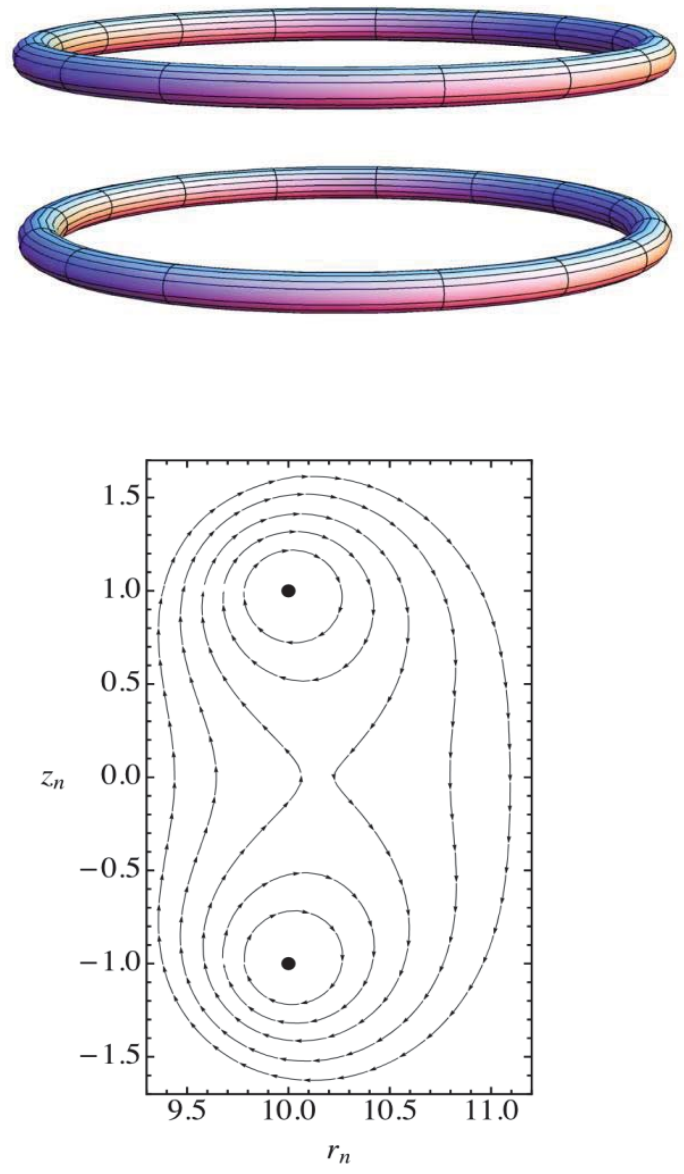

Figure 1: (Top) Visual representation of two levitated coils. (Bottom) Magnetic field lines on the r-z plane associated with the field produced by two coaxial current loops located at $z_{n}= \pm 1$. Each current loop has a normalized radius $a_{n}=10$. Lengths are normalized by $\zeta$, which equals half the distance of separation between the two current loops. Normalized parameters have a subscript $\mathrm{n}$. The null circle is a point at coordinates $\left(r_{n}=10.17, z_{n}=0\right)$.

The large-radius (straight-wire) limit is taken hereafter, and the associated magnetic field lines are illustrated in Fig. 2. The current carried by each wire is the same, and the current $\mathrm{I}$ is in the positive y direction for I > 0. A CTMC simulation has been developed for charged particles that travel within the magnetic field. Each parameter used in the simulation is normalized such that it is dimensionless. A measure of the inaccuracy of a simulated trajectory is the change of a particle's normalized kinetic energy, a quantity that should remain constant because of conservation of energy. The normalized kinetic energy changes typically by less than $0.01 \%$ for each simulated trajectory reported here. Such small changes indicate good computation accuracy is achieved, because the simulation does not employ a conservation of energy equation. Each particle travels a total path length that equals ten times the separation between the wires. Plots of one thousand (1000) simulated classical trajectories are illustrated in Fig. 3. The sign of the charge of a particle is chosen to be positive. However, statistically averaged Monte Carlo results are independent of the sign of charge. All simulated trajectories indicate that the particles remain confined. The results indicate good plasma confinement in the single-particle limit, provided that the average speed of a plasma particle is much smaller than the particle speed in the simulation. The CTMC simulations indicate that the condition for good particle confinement is that the average cyclotron radius, the radius of curvature of the trajectory due to the effect of the local magnetic field, is much smaller than the distance from the magnetic null to one of the current loops, when the local magnetic field is considered to be that at the top of the magnetic well at $x_{n}=0$. 


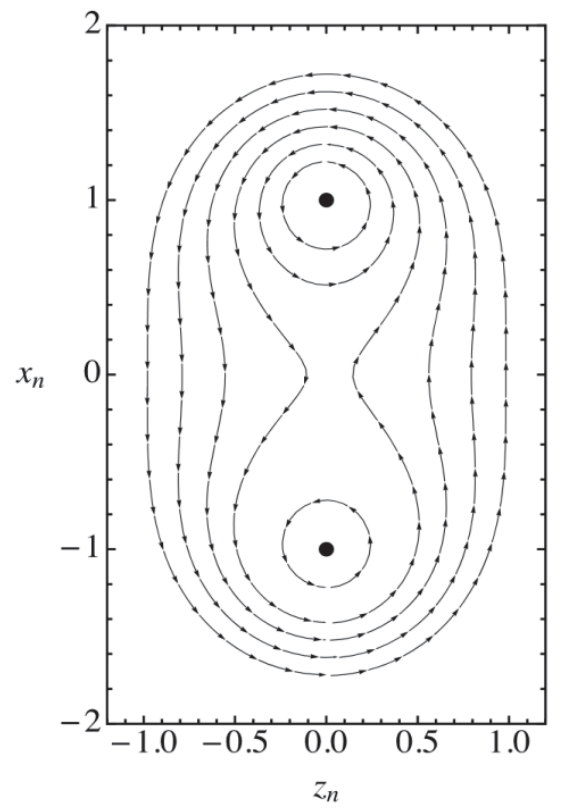

Figure. 2: Magnetic field lines on the $x-z$ plane. The dots represent the locations of two wires that carry equal currents out of the page.

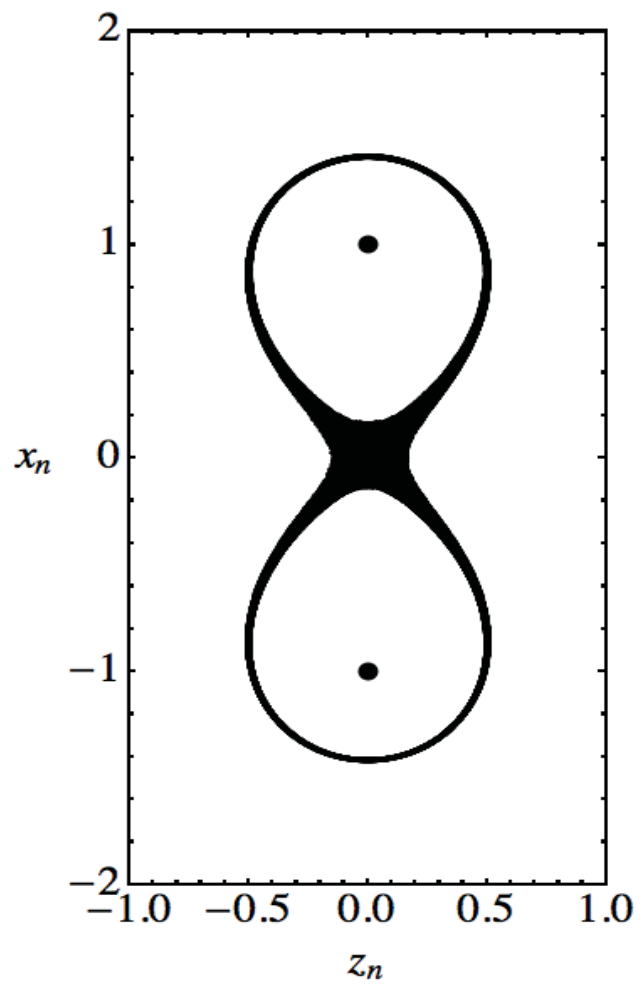

Figure. 3: One thousand (1000) simulated trajectories. The dots represent the locations of two wires that carry equal currents out of the page. Each trajectory start at a randomly chosen position within a box: $0<\mathrm{x}_{\mathrm{n}}<0.1,0<\mathrm{y}_{\mathrm{n}}<0.1,0<\mathrm{z}_{\mathrm{n}}$ $<0.1$. All particles have the same speed, and a randomly chosen initial direction of motion. It is not possible to distinguish between individual trajectories.

\section{LEVITATION OF A PERMANENT RING MAGNET USING HIGH TEMPERATURE SUPERCONDUCTORS}

An experiment has been performed that involves the levitation of a permanent ring magnet using high temperature superconductors. A ring magnet produces a magnetic field that is similar to the field that would be produced by the two coaxial superconducting coils in the far-field limit, except that two cusps are present axially. The similarities and differences are illustrated with Fig. 4. The ring magnet used is an alloy of neodymium, iron, and boron with the chemical formula $\mathrm{Nd}_{2} \mathrm{Fe}_{14} \mathrm{~B}$. The superconductors are bismuth, strontium, calcium, copper, oxide (BSCCO), which is a family of superconductors that is a generalized mixture [12]. There is a numerical notation to describe $\mathrm{BSCCO}$, an example being the material used in this experiment, Bi-2223 [13]. Bi-2223 has a critical temperature of approximately $108 \mathrm{~K}$, so liquid nitrogen cooling is adequate to bring this material to a superconducting state.

The first configuration, which is shown in Fig. 5, was a combination of a large and small BSCCO disc. The neodymium ring magnet (pictured) is stably levitated over the superconductors and is able to rotate freely.

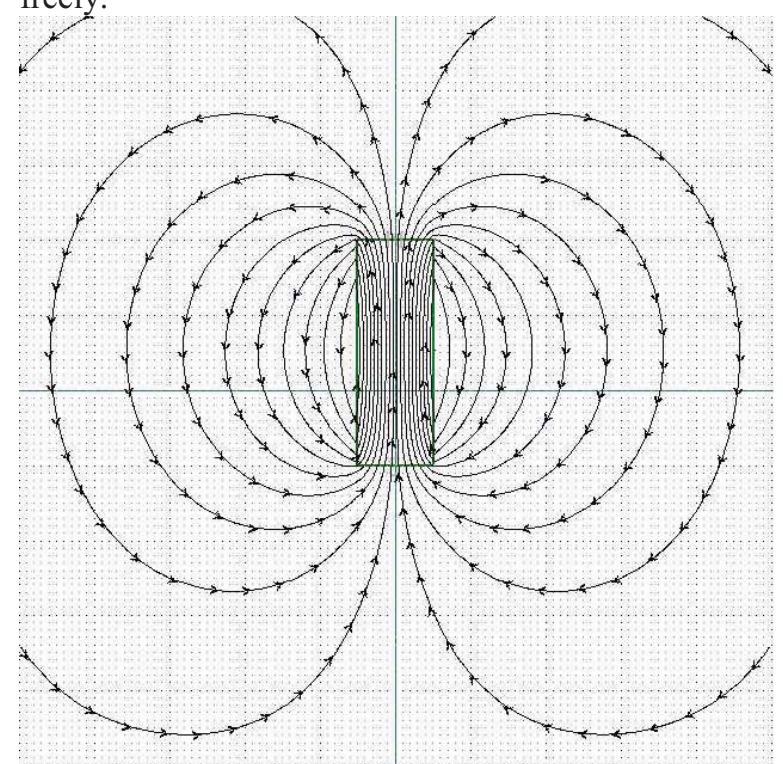

Figure. 4: Magnetic field lines produced by the ring magnet. The illustration demonstrates an axially extended ring magnet in the large radius limit. Vizimag was used to produce this plot. 


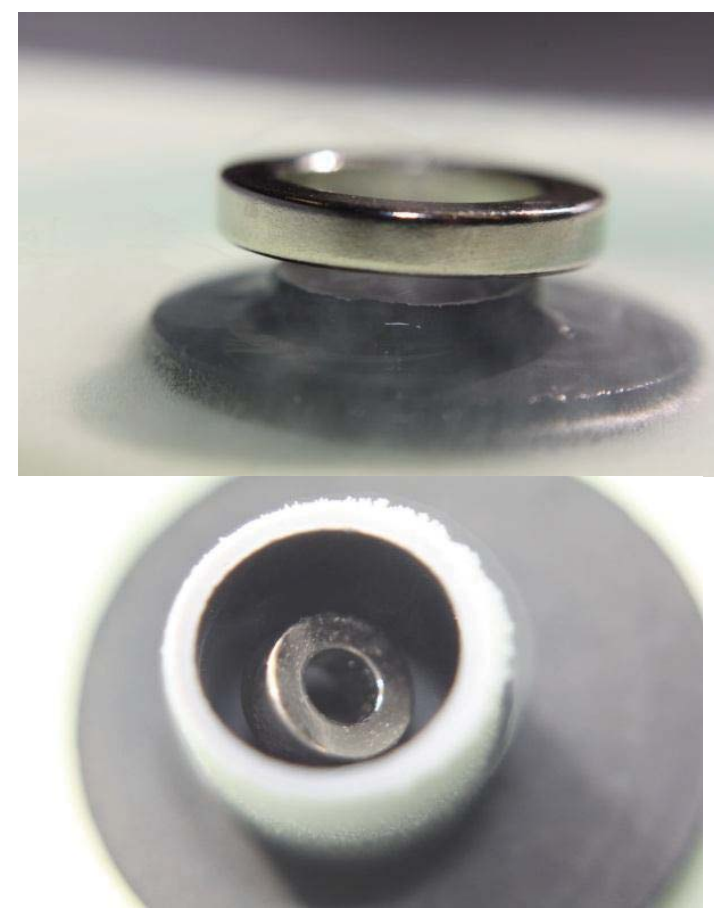

Figure. 5: (Top) Neodymium ring magnet levitated over BSCCO superconductors. (Bottom) Neodymium ring magnet suspended within BSCCO cylinder.

The second "cup" configuration, also shown in Fig. 5, was a combination of a BSCCO disc and a BSCCO cylinder. This configuration demonstrates a more rigid passive feedback system that places the levitated ring magnet within the BSCCO cylinder. There is enough space between the ring magnet and the interior of the cylinder to allow for contactless levitation. When both the BSCCO disc and BSCCO cylinder are superconducting, the magnet is repelled away from both surfaces. When the cylinder is moved around on the disc, the magnet will move with the cylinder without making contact with the disc or cylinder.

There is an issue regarding keeping the plane of the ring horizontal. Over time, the magnet will develop a tilt with respect to the surface of the disc. There are a number of possibilities regarding how this problem can be avoided. One solution involves rotating the ring to give it a moment of inertia strong enough to resist the forces involved in causing the tilt [14]. Two more methods involve active and passive feedback systems. Active feedback would require a circuit that would detect the tilt of the levitated ring, and electromagnets would readjust the tilt of the ring as needed [15]. A passive system includes surrounding the levitated ring with superconducting material [11]. A possible design would be a modified version of the "cup" design (BSCCO disc and cylinder); with the addition of a superconducting rod in the center of the cylinder that threads the center of the ring magnet. The addition of this rod to the design may also be used to keep a pai of levitated coils from tilting with respect to one another.

\section{CONCLUDING REMARKS}

We have studied the possibility of using two levitated magnetic coils as a means of plasma confinement. A classical trajectory Monte Carlo simulation been developed. Plasma confinement has been studied by simulating the motion of particles within the combined magnetic fields of two coaxial current carrying magnetic coils in the large radius limit. An experiment was carried out to study the levitation issues for the two loop system. Although the results are promising, the work reported is preliminary, and further work is warranted to develop a better understanding of the dual levitated coil system.

\section{ACKNOWLEDGMENTS}

The authors would like to thank R. Garrett for helpful comments and suggestions, A. Herro for providing photography assistance, R. Hedlof, and J. Pacheco for help with the illustrations.

This material is based upon work supported by the Department of Energy under Grant No. DE-FG0206ER54883 and by the National Science Foundation under Grant No. PHY-1202428.

\section{REFERENCES}

1. G.B. Andresen et al., Nature (London) 468, 673 (2010).

2. G. Gabrielse et al., Phys. Rev. Lett. 108, 113002 (2012).

3. Y. Enomoto et al., Phys. Rev. Lett. 105, 243401 (2010).

4. C.A. Ordonez and D.L. Weathers, Phys. Plasmas 15, 083504 (2008).

5. A. Zhukovsky, D.T. Garnier, IEEE Transac. Appl. Superconductivity 16, 898 (2006).

6. J.H. Schultz et al., IEEE Transac. Appl. Superconductivity 11, 2004 (2001).

7. A.C. Boxer et al., Nature Phys. 6, 207 (2010).

8. Z. Yoshida et al., Phys. Rev. Lett. 104, 235004 (2010).

9. Y. Yano et al., Fusion Eng. Design 85, 641 (2010).

10. T. Tosaka et al., IEEE Transac. Appl. Superconductivity 17, 1402 (2007).

11. M.Q. Tran, A. Lee and R. Bollens, J. Appl. Phys. 52, 550 (1981).

12. H. Maeda et al., Jpn. J. Appl. Phys. 27, L209 (1988).

13. J.L. Tallon et al., Nature 333,153 (1988). 
14. L.A. Romero, SIAM J. Appl. Math. 63, 2176 (2003).

15. I. Echeverria and M.A. Rubio, Rev. Sci. Instrum. 66, 3931 (1995). 